\title{
Chaos, Determinacy and Fractals in Active-Sterile Neutrino Oscillations in the Early Universe
}

\author{
Kevork N. Abazajiar* and Prateek Agrawa ${ }^{\dagger}$ \\ Department of Physics, University of Maryland, College Park, MD 20742-4111
}

\begin{abstract}
The possibility of light sterile neutrinos allows for the resonant production of lepton number in the early universe through matter-affected neutrino mixing. For a given a mixing of the active and sterile neutrino states it has been found that the lepton number generation process is chaotic and strongly oscillatory. We undertake a new study of this process' sensitivity to initial conditions through the quantum rate equations. We confirm the chaoticity of the process in this solution, and moreover find that the resultant lepton number and the sign of the asymmetry produces a fractal in the parameter space of mass, mixing angle and initial baryon number. This has implications for future searches for sterile neutrinos, where arbitrary high sensitivity could not be determinate in forecasting the lepton number of the universe.
\end{abstract}

PACS numbers: 14.60.Pq; 14.60.St; 26.35.+c; 95.30.-k

\section{INTRODUCTION}

Neutrino physics is entering an era of increasing precision as initial evidence for neutrino oscillations is leading to confirmation and refinement of the neutrino mass and mixing parameters. The solar neutrino deficit is consistent with a matter-affected Mikheyev-SmirnovWolfenstein (MSW) transformation of electron neutrinos, $\nu_{e}$ 1], and was confirmed in the CPT conjugate channel by reactor electron antineutrino disappearance at KamLAND [2]. Atmospheric neutrino disappearance due to apparent neutrino oscillations between what is predominantly $\nu_{\mu}$ and $\nu_{\tau}$ and their CPT conjugates has been confirmed and is being determined precisely by long-baseline neutrino experiments [3, 4].

There were also indications of neutrino oscillations governed by a large mass scale from the short-baseline Liquid Scintillator Neutrino Detector (LSND) [5] that would require a third mass scale difference and therefore a fourth neutrino or more [6. This neutrino could not participate in weak interactions due to the $Z^{0}$ width 7 , and therefore is dubbed "sterile." Sterile neutrinos are prevalent in most models of neutrino mass generation mechanisms [8], and could be light enough to participate in neutrino oscillations. The four neutrino oscillation hypothesis for the LSND result is in conflict with new results from the from the short-baseline MiniBOONE experiment [9]. The parameter space of interest for the LSND and MiniBOONE results are a small fraction of that of interest for lepton number generation, and do not exclude the possibility of this mechanism. Moreover, such light sterile neutrinos in the parameter space of interest for lepton number generation, and outside of the MiniBooNE bounds are favored to produce the electron fraction required by successful $r$-process nucleosynthesis in Type-II supernovae [10, 11].

*Electronic address: kev@umd.edu

${ }^{\dagger}$ Electronic address: $\overline{\text { apr@umd.edu }}$
See-saw type mass generation mechanisms could readily include such light sterile neutrinos 12 .

The effects of sterile neutrinos in the early universe have been under study for some time. Early work studied the effect of the partial or complete thermalization of the sterile neutrino through oscillations, which would alter the energy density and expansion rate of the universe prior to primordial nucleosynthesis, and therefore the production of the light elements [13, 14, 15, 16]. The primordial helium yield is very sensitive to the expansion rate, and has been used to place bounds on four neutrino models with appreciable mixing between active and sterile neutrinos of the kind required for the LSND indications 17, 18. It was also discovered that an MSW resonance can cause the generation of a large neutrino asymmetry that could modify primordial nucleosynthesis even with very small mixing angles between the active and sterile neutrinos [19. This was confirmed in Ref. [20, and further found rapid oscillations in the asymmetry generation, extreme sensitivity to initial conditions, and chaoticity in certain regions of parameter space of mass and mixing angle.

A key question regarding the chaoticity of the lepton generation mechanism is the determinacy of the sign of the asymmetry given a mixing angle and mass-splitting for the active-sterile neutrino pair. That is, if a mass and mixing angle were experimentally measured for the pair, could the sign and magnitude of the cosmological neutrino asymmetry be predicted? One way of assessing the predictability of the final lepton number given a potentially experimentally determined mass and mixing angle is the level of information loss in the lepton number generation process. The level of information loss in the chaotic process was explored in Ref. [21, which found appreciable loss of initial conditions due to chaoticity in small regions of parameter space. The sensitivity of the sign and amplitude of the asymmetry to the mixing parameter space was found to be "complex" in Ref. 22.

All of the work discussed thus far was done through the quantum rate equations (QRE's), where the neutrino 
system is modeled to follow the average neutrino momentum $\langle p\rangle \approx 3.15 T$. The strong momentum dependence of the resonant process of lepton number generation suggests that an accurate solution should take into account the full spectrum in the solution of the equations through the so-called Quantum Kinetic Equations (QKE's). The narrowness of the resonance requires exceptionally fine momentum binning and therefore following the exponential growth in a very large number of momenta modes. Several authors found limitations to solving the equations numerically [23, 24]. For example, Ref. 23] found oscillatory behavior in a range of parameter space with the QKE's, but could not conclude that it was physical and not numerical. The QKE's were solved through the oscillatory phase by Ref. [25, who used a new binning procedure following the resonance to simulaneously have fine binning yet a manageable number of momenta modes. They found that the chaotic nature of the generation process remained.

In this work, we explore the nature of the lepton number generation process and its chaoticity. We confirm that the resonant generation is highly oscillatory in regions of the parameter space and extremely sensitive to the initial conditions. Moreover, we explore the dependence of the final sign of the asymmetry on the oscillation parameter space and find that the extreme sensitivity to the parameter space gives rise to a fractal structure in the final sign dependence on mass-splitting and mixing angle. This has significant implications for the determinacy of the cosmological lepton number in the case of discovering a small sterile neutrino mixing with the active neutrino sector. If the parameters are found to lie in a region of fractal nature, even arbitrarily high precision in the determination of the parameters will not allow for a prediction of the cosmological lepton number.

We will briefly introduce our system in Sec. II. including the effects of our constant momentum approximation. We go on in Sec. III to present the solution under the above approximation, where we show that the final sign of the asymmetry is not just chaotic, but exhibits a self-similarity like that of a fractal at very small scales. We compare our results with Ref. 22, which shows that the resolution and numerical error are responsible for the seeming "white noise" structures in the parameter space. We conclude in Sec. IV.

\section{THE QUANTUM KINETIC AND RATE EQUATIONS}

For specificity, we concentrate on oscillations between the $\tau$-neutrino $\left(\nu_{\tau}\right)$ and a sterile neurino $\left(\nu_{s}\right)$. The vacuum mixing angle is defined by the following equation,

$$
\left[\begin{array}{l}
\nu_{\tau} \\
\nu_{s}
\end{array}\right]=\left[\begin{array}{cc}
\cos \left(\theta_{0}\right) & \sin \left(\theta_{0}\right) \\
-\sin \left(\theta_{0}\right) & \cos \left(\theta_{0}\right)
\end{array}\right]\left[\begin{array}{l}
\nu_{a} \\
\nu_{b}
\end{array}\right]
$$

where $\nu_{a}$ and $\nu_{b}$ are mass eigenstates. Conventionally, $\cos (\theta)$ is chosen to be positive, and $\delta m^{2} \equiv m_{b}^{2}-m_{a}^{2}$.
An $\alpha$-type neutrino asymmetry is defined as

$$
L_{\nu_{\alpha}} \equiv \frac{n_{\nu_{\alpha}}-n_{\bar{\nu}_{\alpha}}}{n_{\gamma}}
$$

where the photon number density $n_{\gamma}=2 \zeta(3) T^{3} / \pi^{2}$. The effective total lepton number $L^{(\alpha)}$, useful when defining the evolution equations below, is given by

$$
L^{(\alpha)}=L_{\nu_{\alpha}}+L_{\nu_{e}}+L_{\nu_{\mu}}+L_{\nu_{\tau}}+\eta
$$

where $\eta \equiv n_{\mathrm{b}} / n_{\gamma}$ is the baryon to photon ratio at the epoch of oscillations.

The density matrix parametrization for an activesterile oscillation can be chosen as

$$
\begin{aligned}
\rho_{\tau \beta^{\prime}}(p) & =\frac{1}{2}\left[P_{0}(p) I+\mathbf{P}(p) \cdot \sigma\right] \\
\bar{\rho}_{\tau \beta^{\prime}}(p) & =\frac{1}{2}\left[\bar{P}_{0}(p) I+\overline{\mathbf{P}}(p) \cdot \sigma\right]
\end{aligned}
$$

where $\mathbf{P}(p)=P_{x}(p) \hat{\mathbf{x}}+P_{y} \hat{\mathbf{y}}+P_{z} \hat{\mathbf{z}}$ is the polarization vector; $\sigma=\sigma_{x} \hat{\mathbf{x}}+\sigma_{y} \hat{\mathbf{y}}+\sigma_{z} \hat{\mathbf{z}}$ are the usual Pauli matrices. The evolution of $\mathbf{P}(p), P_{0}(p), \overline{\mathbf{P}}(p)$ and $\bar{P}_{0}(p)$ is given by the following equations 25 ,

$$
\begin{aligned}
\frac{d \mathbf{P}}{d t} & \left.=\mathbf{V}(p) \times \mathbf{P}(p)-D(p)\left[P_{x}(p) \hat{\mathbf{x}}+P_{y}(p)\right] \hat{\mathbf{y}}\right]+\frac{d P_{0}}{d t} \hat{\mathbf{z}} \\
\frac{d P_{0}}{d t} & \simeq \Gamma(p)\left[\frac{f_{e q}(p)}{f_{0}(p)}-\frac{1}{2}\left(P_{0}(p)+P_{z}(p)\right)\right] .
\end{aligned}
$$

The equations for the anti-particles are given by the obvious substitutions, $\mathbf{P} \rightarrow \overline{\mathbf{P}}, P_{0} \rightarrow \bar{P}_{0}, \mathbf{V}(p) \rightarrow \overline{\mathbf{V}}(p)$, $f_{e q}(p) \rightarrow \bar{f}_{e q}(p) . \overline{\mathbf{V}}(p)$ is obtained by replacing $L^{(\tau)}$ by $-L^{(\tau)}$. The total collision rates of the flavor neutrino and anti-neutrino are approximately equal, $\Gamma(p) \simeq \bar{\Gamma}(p)$. These 8 equations form the quantum kinetic equations for active-sterile oscillation.

The damping coefficient is given by

$$
D(p)=\Gamma(p) / 2,
$$

where $\Gamma(p)$ is the total collision rate of the neutrino, given by $0.92 G_{F}^{2} T^{5} p$ for the $\tau$-neutrino. Further, $\bar{D} \simeq D$ to a good approximation [22].

The rotation vector $\mathbf{V}(p)$ has the following components

$$
\begin{aligned}
V_{x}(p) & =\frac{\delta m^{2}}{2 p} \sin 2 \theta_{0} \\
V_{y}(p) & =0 \\
V_{z}(p) & =V_{0}(p)+V_{L}(p),
\end{aligned}
$$

where

$$
\begin{aligned}
V_{0}(p) & =-\frac{\delta m^{2}}{2 p} \cos 2 \theta_{0}+V_{1} \\
V_{1}(p) & =-\frac{7 \sqrt{2}}{2} \frac{\zeta(4)}{\zeta(3)} \frac{G_{F}}{M_{z}^{2}} n_{\gamma} p T\left[n_{\nu_{\tau}}+n_{\nu_{\bar{\tau}}}\right] \\
V_{L}(p) & =\sqrt{2} G_{F} n_{\gamma} L^{(\tau)}
\end{aligned}
$$


Here $n_{\gamma}$ is the photon equilibrium number density, $n_{\nu_{\tau}}$ and $n_{\nu_{\bar{\tau}}}$ are normalized to unity and $f_{e q}(p)$ is the Fermi-Dirac distribution with a chemical potential $\mu_{\tau}$.

The chaotic nature of the solution is seen to not depend on $\eta$, which was set to $\sim 1 \times 10^{-10}$ (though the sign of the asymmetry can be sensitive to this initial value as well). The well-known Fermi-Dirac distributions are

$$
\begin{aligned}
f_{0}(p) & =\frac{1}{1+\exp (p / T)} \\
f_{e q}(p) & =\frac{1}{1+\exp \left(p / T-\tilde{\mu}_{\tau}\right)},
\end{aligned}
$$

where $\tilde{\mu}_{\tau} \equiv \mu_{\nu_{\tau}} / T$. To get the distribution for antineutrinos, we substitute $\tilde{\mu}_{\tau} \rightarrow \tilde{\mu}_{\bar{\tau}}$. The chemical potentials can be found using the neutrino asymmetry. For a system in thermal equilibrium, the asymmetry is given by

$$
L_{\nu_{\tau}}=\frac{1}{4 \zeta(3)} \int_{0}^{\infty} \frac{x^{2} d x}{1+e^{x-\tilde{\mu}_{\tau}}}-\frac{1}{4 \zeta(3)} \int_{0}^{\infty} \frac{x^{2} d x}{1+e^{x-\tilde{\mu}_{\bar{\tau}}}} .
$$

We can expand the above integral in $\left(\tilde{\mu}_{\tau}-\tilde{\mu}_{\bar{\tau}}\right)$

$$
\begin{aligned}
L_{\nu_{\tau}} \simeq & \frac{1}{24 \zeta(3)}\left[\pi^{2}\left(\tilde{\mu}_{\tau}-\tilde{\mu}_{\bar{\tau}}\right)\right. \\
& \left.+6\left(\tilde{\mu}_{\tau}^{2}-\tilde{\mu}_{\bar{\tau}}^{2}\right) \ln (2)+\left(\tilde{\mu}_{\tau}^{3}-\tilde{\mu}_{\bar{\tau}}^{3}\right)\right] .
\end{aligned}
$$

We need another relation between $\tilde{\mu}_{\tau}$ and $\tilde{\mu}_{\bar{\tau}}$ to solve for them. For temperatures greater than the chemical decoupling temperatures, pair creation and annihilation processes are fast enough to ensure chemical equilibrium. However, below these temperatures, neutrinos no longer remain in thermal equilibrium, though weak interactions are fast enough to thermalize them. As a consequence, the value of $\tilde{\mu}_{\tau}$ remains fixed at the decoupling temperature, but $\tilde{\mu}_{\bar{\tau}}$ continues to decrease. In summary

$$
\begin{array}{rl}
\tilde{\mu}_{\tau}+\tilde{\mu}_{\bar{\tau}}=0 & T \gtrsim T_{d e c}^{\tau} \\
\tilde{\mu}_{\tau}=\tilde{\mu}_{\tau}\left(T=T_{d e c}^{\tau}\right) & T \lesssim T_{d e c}^{\tau},
\end{array}
$$

where $T_{d e c}^{\tau} \simeq 3.5 \mathrm{MeV}$. These two equations above determine the chemical potentials to be used in the FermiDirac distributions.

We employ the approximation that all the neutrinos have the same momentum, viz. $p=\langle p\rangle \approx 3.15 T$ for our analysis. The approximation inflates the rate at which neutrino asymmetry is created and therefore may be unsuitable for the thermal regions where the asymmetry growth is shown to be exponential [23]. Apart from this shortcoming, this approximation has the significant advantage of being numerically tenable, and is useful to explore the general features of the system. We also carried out analysis using the full momentum dependent quantum kinetic equations (QKEs). The full system is too numerically intensive to run over the entire parameter range at high resolution to study the fractal structure. Moreover, our work with the full QKEs never reached

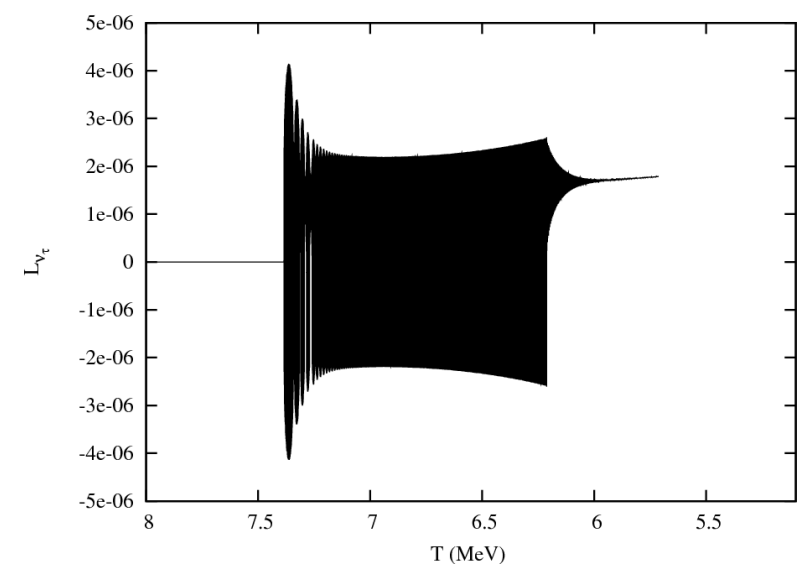

FIG. 1: Shown is the evolution of the lepton number over temperature for a particular case of $\nu_{\tau} \leftrightarrow \nu_{s}$ mixing, $\delta m^{2}=$ $-0.01 \mathrm{eV}, \sin ^{2} 2 \theta=2 \times 10^{-5}$.

the numerical stability which we required, and describe below.

Further, following the work of Ref. [22, we approximate $P_{0}$ to be a constant, set to 1 . The asymmetry is given simply by

$$
L_{\nu_{\tau}}=\frac{3}{8}\left(P_{z}-\bar{P}_{z}\right) .
$$

In order to achieve numerical efficiency and stability, we rewrite $\left\{P_{i}, \bar{P}_{i}\right\}$ in the above equation as $\left\{P_{i}^{+}, P_{i}^{-}\right\}$, where $P_{i}^{ \pm}=P_{i} \pm \bar{P}_{i}$. This separates out the large quantities and the small quantities in the differential equation [22]. Thus, the equations take the form

$$
\begin{aligned}
& \dot{P}_{x}^{+}=-D P_{x}^{+}-V_{0} P_{y}^{+}-V_{L} P_{y}^{-} \\
& \dot{P}_{y}^{+}=-D P_{y}^{+}-V_{x} P_{z}^{+}+V_{0} P_{x}^{+}+V_{L} P_{x}^{-} \\
& \dot{P}_{z}^{+}=V_{x} P_{y}^{+} \\
& \dot{P}_{x}^{-}=-D P_{x}^{-}-V_{0} P_{y}^{-}-V_{L} P_{y}^{+} \\
& \dot{P}_{y}^{-}=-D P_{y}^{-}-V_{x} P_{z}-V_{0} P_{x}^{-}+V_{L} P_{x}^{+} \\
& \dot{P}_{z}^{-}=V_{x} P_{y}^{-} .
\end{aligned}
$$

These are coupled non-linear differential equations, with no analytical solution. The system is seen to undergo resonance at $V_{0}=0$, if $\delta m^{2}<0$. The asymmetry $L_{\nu_{\tau}}$ undergoes rapid oscillation at the resonance, finally settling down to a power-law-like growth. See Fig. 1 for an example of the oscillatory evolution. The magnitude of the final asymmetry is given by the solution of $V_{L}+V_{0}=0$, and is given by $\left|L_{\nu_{\tau}}\right| \simeq$ $11\left(\left|\delta m^{2}\right| / \mathrm{eV}^{2}\right)(\mathrm{MeV} / T)^{4}$. After resonance, $L_{\nu_{\tau}}=0$ becomes an unstable extremum, and the system is trapped into one of the two minima given above. A detailed discussion of how the asymmetry evolves can be found in Ref. 22]. The final sign of the asymmetry, however, is not fixed. It is seen to be very sensitive to initial conditions, mixing parameters, even choice of step sizes and integrators 26]. 

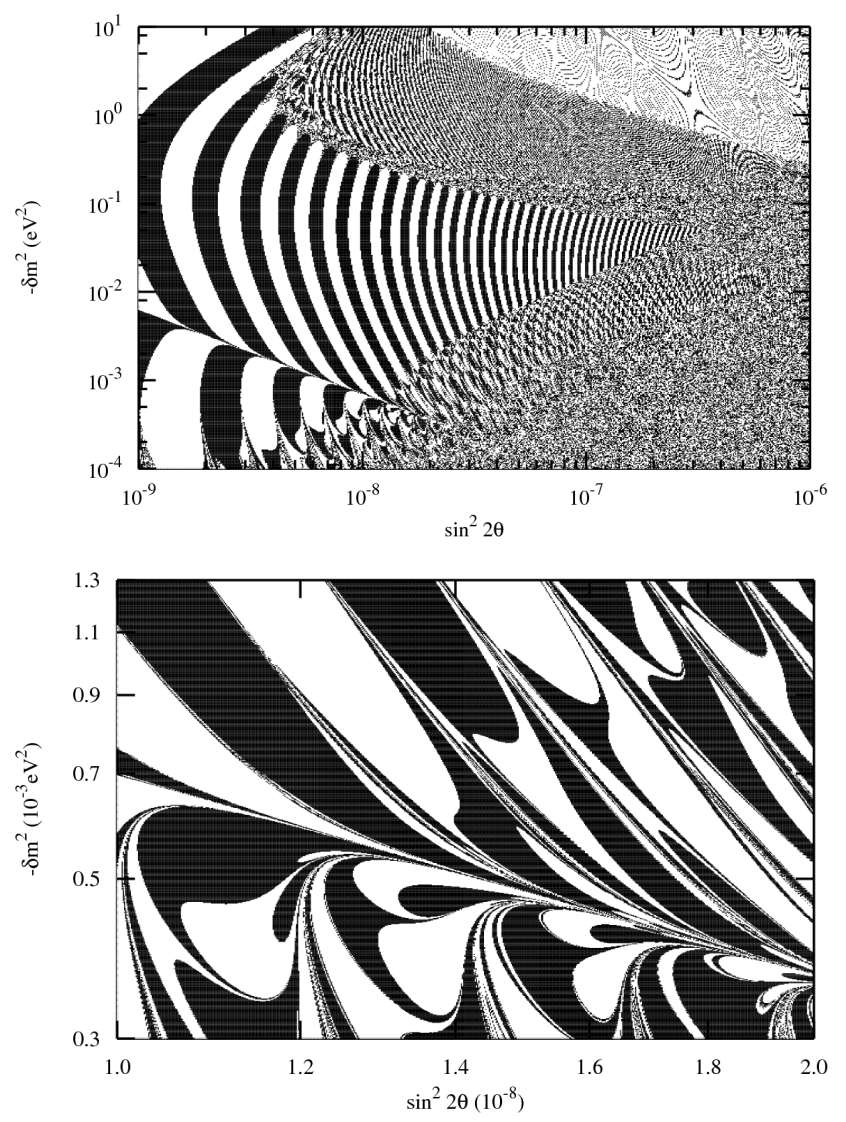

FIG. 2: Shown is the final sign of the lepton asymmetry as a function of mass splitting and mixing angle in the case of $\nu_{\tau} \leftrightarrow \nu_{s}$ mixing for a wide range of parameter space (top), and a zoomed region (bottom). White indicates a positive sign and black negative.

The differential equations (Eq. 5) are expressed in terms of time-derivatives but from a cosmological point of view, we are interested to look at the solutions in terms of temperature. The time-temperature relation is given by $d t / d T \simeq-M_{\mathrm{pl}} / 5.44 T^{3}, M_{\mathrm{pl}}$ being the Planck mass.

\section{RESULTS}

We plot the asymmetry as a function of temperature for a couple of parameter sets, and our results match up well with previous work 22 . When we scan the entire parameter range shown in Fig. 2, we note some interesting facts. First, in our calculations, the band-structure seems to continue up to many more bands as compared to seen in Ref. 22. Further, we can make out structures even beyond the primary band structure, which has previously been described as pure white noise. Our numerical routines show that the number of oscillations increases as the value of $\theta_{0}$ increases, and hence the number of steps for the evolution of the differential equations increases. This requires that the absolute error per step in the differential equation be very small in order for the final sign to
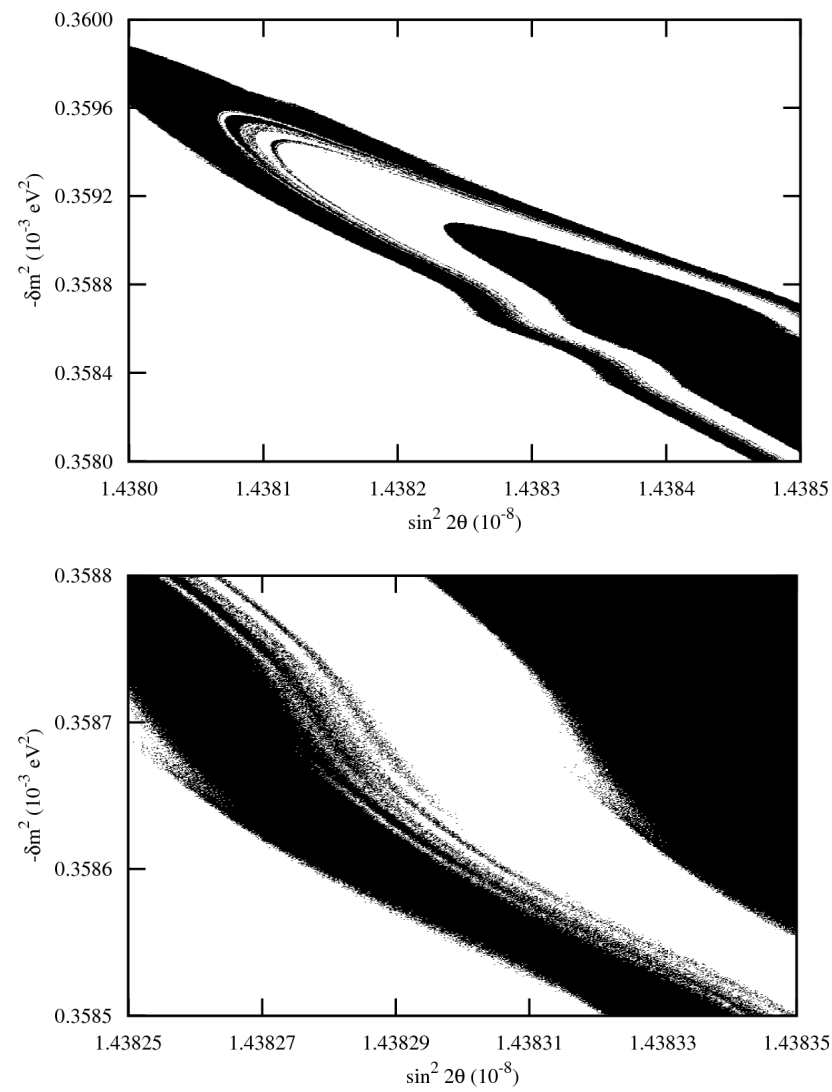

FIG. 3: Shown is the final sign of the lepton asymmetry as a function of mass splitting and mixing angle in a zoomed parameter range to show detail of the sign dependence.

be numerically stable. Also, beyond the primary bands, the structure becomes very fine, and an analysis with a coarse resolution will just sample the fine structure as an apparent random sign distribution.

To resolve the fine structure, we zoom into a part of the parameter space. We see that the region which shows no interesting features in entire range, shows definite structure when we improve the resolution in Fig. 3. with further zoom in to the edge of the bands seen in figure. The edge is seen to have structure at very high zoom magnitudes. This behavior is replicated when we zoom into other parameter ranges. This suggests that the sign of asymmetry exhibits the characteristics of a fractal with respect to mixing parameters.

We employed the Gnu Scientific Library extensively for our numerical analysis ${ }^{1}$. The system of differential equations are extremely sensitive numerically, and therefore demand a very careful treatment. We use the adaptive Runge-Kutta-Fehlberg routine for our system. For the momentum averaged solutions, we use a very stringent error bound per step of evolution, so that the accumu-

\footnotetext{
${ }^{1}$ http://www.gnu.org/software/gsl/
} 
lated error does not affect the final sign of asymmetry. We check that our results converge for a more stringent error bound. As $\theta_{0}$ and $-\delta m^{2}$ increase, so does the number of resonant oscillations. This in turn implies that the step size would decrease in order to follow the resonance correctly, giving us a larger number of steps overall. We kept our error bound near $1 \times 10^{-15}$, and we found that the error begins to dominate the solution around $1 \times 10^{-10}$. This may be why we see significantly more structure in Fig. 2 than what was previously seen, in addition to the high resolution sampling of the parameter space.

To characterize the nature of the fractal solutions, we estimate its fractal dimension with the correlation integral

$$
C(\epsilon)=\lim _{N \rightarrow \infty} \frac{1}{N^{2}} \sum_{i, j=1}^{N} \Theta\left(\epsilon-\left\|\vec{x}_{i}-\vec{x}_{j}\right\|\right),
$$

where $\vec{x}_{i}$ are the positions of the positive sign lepton number in parameter space and $\Theta(x)$ is the Heaviside function. For finite $N$ the integral is the correlation sum. For fractal structures, at high $N$ the sum tends to

$$
C(\epsilon) \sim \epsilon^{\alpha},
$$

where $\alpha$ is the correlation dimension, an approximation of the fractal dimension. For the range $-0.003 \mathrm{eV}^{2}<$ $\delta m^{2}<-0.001 \mathrm{eV}^{2}, 10^{-8}<\sin ^{2}(2 \theta)<2 \times 10^{-8}$ we find a power-law dependence of the correlation sum, and arrive at a convergence of the correlation dimension of approximately 1.05 for $N \gtrsim 10^{4}$. For larger mixing angles, we obtain higher correlation dimensions of 1.35 .

\section{CONCLUSION}

We have explored the generation of cosmological lepton number in the case of the presence of a light sterile neutrino state with small mixing with an active neutrino. Consistent with previous studies of lepton number generation using the momentum averaged quantum rate equations, we find that the process is chaotic and highly sensitive to the mixing parameters coupling the active and sterile neutrino states.

Our main results are the fractal nature of the dependence on the sign of the lepton number to the mixing parameters as well as the initial baryon number. This leaves significant implications for the predictability of the lepton number of the universe, even in the case of arbitrarily precise determinations of the mixing parameters between the active and sterile states in certain regions of the parameter space. Future searches for subdominant mixings with the active neutrinos and unitarity in the active neutrino mixing matrix therefore could be indeterminant in predicting the sign and magnitude of the cosmological lepton number.

The fractal or correlation dimension depends on the region in parameter space that is examined, which is expected. The banded structure of the sign of the parameter space undergoes changes in its structure throughout the space, with some definitive lines in the pattern change. The fractal dimension therefore shifts in the parameter space. This is likely due to the fact that the frequency of the rapid oscillations are deterimined by the oscillation dynamics, and the point at which they stop or freeze-out is determined by the expansion dynamics, which depend on the total radiation energy density of the universe independent of the neutrino physics.

Our work has focused on the single-momentum quantum rate equations for the solution of this system, and we acknowledge that it has been shown that the behavior changes in the full momentum-dependent quantum kinetic description. Studies have found that the oscillatory behavior persists and may be chaotic in the full quantum kinetic equation solution [23, 25]. Since there are numerical difficulties in quantifying the nature of the solution in the full momentum case, which we find in our own work, the resolution of this question may require a completely new formulation of the quantum kinetic equations and their solution in order to make them numerically stable to provide a convincing solution of the evolution. The work presented here highlights the new type of chaotic, fractal physics that can arise in this system, and its consequences in determining the lepton number of the universe given the existence of a light sterile neutrino. If a light sterile neutrino is discovered within this parameter space, a new approaches to quantifying the lepton number of the universe will be strongly motivated. Though if the sign of the lepton produces such fractal structure, the sign of the lepton asymmetry may be indeterminate due to arbitrarily fine fractal structures.

\section{Acknowledgments}

We thank John Beacom and Nicole Bell for discussions in very early stages of this work. We also thank Edward Ott for useful discussions. This work was supported at the University of Maryland by the Maryland Center for Fundamental Physics.
[1] S. N. Ahmed et al. (SNO), Phys. Rev. Lett. 92, 181301 (2004), nucl-ex/0309004.

[2] T. Araki et al. (KamLAND), Phys. Rev. Lett. 94, 081801
(2005), hep-ex/0406035.

[3] D. G. Michael et al. (MINOS), Phys. Rev. Lett. 97, 191801 (2006), hep-ex/0607088. 
[4] M. H. Ahn et al. (K2K), Phys. Rev. D74, 072003 (2006), hep-ex/0606032.

[5] C. Athanassopoulos et al. (LSND), Phys. Rev. Lett. 81, 1774 (1998), nucl-ex/9709006.

[6] M. Sorel, J. M. Conrad, and M. Shaevitz, Phys. Rev. D70, 073004 (2004), hep-ph/0305255.

[7] W. M. Yao et al. (Particle Data Group), J. Phys. G33, 1 (2006).

[8] R. N. Mohapatra and A. Y. Smirnov, Ann. Rev. Nucl. Part. Sci. 56, 569 (2006), hep-ph/0603118.

[9] T. M. Collaboration, Physical Review Letters 98, 231801 (2007).

[10] G. C. McLaughlin, J. M. Fetter, A. B. Balantekin, and G. M. Fuller, Phys. Rev. C59, 2873 (1999), astro$\mathrm{ph} / 9902106$.

[11] J. Fetter, G. C. McLaughlin, A. B. Balantekin, and G. M. Fuller, Astropart. Phys. 18, 433 (2003), hep-ph/0205029.

[12] A. de Gouvea, Phys. Rev. D72, 033005 (2005), hep$\mathrm{ph} / 0501039$.

[13] P. Langacker (1989), uPR-0401T.

[14] R. Barbieri and A. Dolgov, Phys. Lett. B237, 440 (1990).

[15] K. Kainulainen, Phys. Lett. B244, 191 (1990).
[16] X. Shi, D. N. Schramm, and B. D. Fields, Phys. Rev. D48, 2563 (1993), astro-ph/9307027.

[17] P. Di Bari, Phys. Rev. D65, 043509 (2002), [Addendumibid. D 67, 127301 (2003)], hep-ph/0108182.

[18] K. N. Abazajian, Astropart. Phys. 19, 303 (2003), astro$\mathrm{ph} / 0205238$.

[19] R. Foot, M. J. Thomson, and R. R. Volkas, Phys. Rev. D53, 5349 (1996), hep-ph/9509327.

[20] X.-D. Shi, Phys. Rev. D54, 2753 (1996), astro$\mathrm{ph} / 9602135$.

[21] P.-E. N. Braad and S. Hannestad (2000), hep$\mathrm{ph} / 0012194$.

[22] K. Enqvist, K. Kainulainen, and A. Sorri, Physics Letters B 464, 199 (1999).

[23] P. Di Bari and R. Foot, Physical Review D 61, 105012 (2000).

[24] R. R. Volkas and Y. Y. Y. Wong, Phys. Rev. D62, 093024 (2000), hep-ph/0007185.

[25] K. Kainulainen and A. Sorri, JHEP 0202, 020 (2002).

[26] X. Shi, Physical Review D 54, 2753 (1996). 